\title{
Attention switching between perception and memory
}

\author{
ROBERT J. WEBER, DIANA BYRD BURT, and NICHOLAS C. NOLL \\ Oklahoma State University, Stillwater, Oklahoma
}

\begin{abstract}
Two experiments were conducted to explore the switching of attention between perception and memory. In Experiment 1, college students performed a task that required them to cycle or switch attention between perceptual and memorial inputs. Switching times of 293 and $376 \mathrm{msec}$ per switch were obtained on the basis of two formulas. In Experiment 2, the attentional load was manipulated by varying the number of perceptual and memorial inputs. Switching time increased as a function of list length, indicating that item load affects both the control processes that set attentional allocation policies and one's ability to perform memory and/or perceptual tasks. These results suggest that modularity, or encapsulation of item and control-process systems, does not hold. A model is presented that depicts the relation between item and control-process representation in rapidly alternating attention between perception and memory.
\end{abstract}

Many common tasks, such as reading (Baddeley, 1982; Byrd \& Gholson, 1984) and using a memorized shopping list, seem to involve a rapid cycling of attention or switching between information available in the environment and information stored in memory. The nature of switching between perception and memory is the concern of the present study.

In the past, typical methods used to study attention switching involved either dichotic listening, in the classical tradition of Broadbent (1958), or the manipulation of expectancies as to the spatial location of critical events (Shulman, Remington, \& McLean, 1978). In the dichotic listening task, each ear concurrently receives a different set of digits to be recalled. The pattern of recall does not typically follow the order of presentation, but is first by one ear and then the other, presumably because of the difficulty or time required to alternate attention between ears. In the expectancy manipulation approach, the probable location of a target is cued, and then the target is presented. In cases of agreement between cued and real target information, there is a savings in reaction time over a control condition with a noninformative cue. In cases of disagreement between cue and target location, there is a substantial increase in processing over a control condition. This latter difference could be interpreted as a switching time.

However excellent the dichotic and expectancy methods may be for the study of certain aspects of attention, they may not be entirely appropriate for the study of rapid selfpaced switches between perception and memory. The

The first author would like to acknowledge the support and use of facilities from the Department of Psychology and Social Relations, Harvard University, during his sabbatical leave. D. B. Burt is now at the University of Texas Mental Sciences Institute, 1300 Moursund, Houston, TX 77030. Requests for reprints should be sent to R. J. Weber, Department of Psychology, Oklahoma State University, Stillwater, OK 74078.
Broadbent method does not allow for direct timing of switching processes. Switching time could only be estimated indirectly by varying stimulus presentation rates. The shortest interstimulus interval (i.e., time between stimuli presented to alternate ears) at which subjects could accurately recall items would be an indirect measure of switching time. In contrast, the expectancy approach is a discrete trials probabilistic approach, and it allows for fairly direct timing of one switching process. However, it only provides an estimation of single attention switches. It may not reflect, therefore, the cycle of loading and flushing of buffers that occurs in a continuous task. What seems to be required is a procedure that is rapid and selfpaced, continuous, and deterministic.

List processing is a procedure that satisfies these requirements (Jersild, 1927; Spector \& Biederman, 1976; Weber, Blagowsky, \& Mankin, 1982). In a typical experiment (Jersild, 1927), subjects read through a list that contained either words or two-digit numbers. The subjects were to respond to stimuli that were words by saying words opposite in meaning (e.g., day-night); they were to respond to stimuli that were digit pairs by subtracting 3 from each number. A mixed list consisted of both words and numbers. The time to read mixed lists was compared with time to read pure lists, consisting of words only or digit pairs only. The difference in production time between mixed and pure lists was considered a measure of the time it took to switch response outputs (Jersild, 1927).

Recently, Weber et al. (1982) used switching-time procedures to establish a model of the internal representational processes involved in switching speech-output intensities. In particular, they were concerned (Experiments 3 and 4) with the nature of intensity representation in speech production. They contrasted two models. The first was an analog model in which a selection pointer moved along an internal intensity continuum. This model 
predicted that the farther apart two intensities are, the longer the switching time for alternating between them. A further implication is that switching times should be additive. For example, for three increasing intensities, $a$, $b$, and $c$, the corresponding switching times should be additive, such that $a b+b c=a c$. The second model tested was a symbolic model in which an intensity value was fed as an argument into an intensity generator function. This model of control processes and intensity representation predicted equal switching times irrespective of the switching interval, with $a b=b c=a c$. In fact, a symbolic model was supported. That is, switching time did not differ as a function of intensity differences.

In the present experiments, list processing and switching procedures were also used to investigate control processes. The control processes involved, however, were those used to switch attention between perception and memory. This type of attention switching merits study for several reasons. First, as mentioned previously, perception-memory switches are apparently involved in many common processes, such as reading. Second, the proposed attention-switching methodology has not been used, to our knowledge, to study attention switches between different list-input modalities.

In Experiment 1, basic procedures were established to study perception-memory attention switching. In Experiment 2 , several alternative ways of representing items and control processes were studied. The issue was whether items held in short-term memory and the control processes involved in attention switching share capacity or whether they are independent of one another. Finally, a model is proposed that depicts the relation between item and control-process representation in perception-memory attention switching.

\section{EXPERIMENT 1}

The purpose of this experiment was to develop a basic switching-time procedure for cycling between perceptual and memorial input. Such a procedure should allow for comparing the time it takes to process information from memory and from perception to the time it takes to process information by alternating between perceptual and memorial inputs. Switching time was investigated in a paradigm that involved cycling between two different inputs, perceptual and memorial information. The basic paradigm used to study perception-memory switching is shown in Table 1. In the memory condition, a list of items in Display 1 is presented (e.g., BHD), the items are committed to memory, and the display terminates. As soon as Display 2 (a row of asterisks) is presented, the names of the items are to be spoken as rapidly as possible. The primary response measure is the production time between onset of Display 2 and completion of naming the items. In the perception condition, a row of asterisks appears in Display 1 (signifying an empty memory set). Another list of items (e.g., RNF) is made available in the perceptual field with Display 2, which the subject is to read aloud
Table 1

\begin{tabular}{clll}
\multicolumn{5}{c}{ Processing } & Modes: Example Trials & \\
\hline Length & Condition & Display 1/Display 2 & Response \\
\hline \multirow{3}{*}{3} & Memory & BHD/*** & BHD \\
& Perception & ***/RNF & RNF \\
& Alternate & BHD/RNF & RBNHFD \\
6 & Memory & BHDRNF/****** & BHDRNF \\
& Perception & $* * * * * /$ BHDRNF & BHDRNF \\
\hline
\end{tabular}

as rapidly as possible. In the alternate condition, the subject alternates as rapidly as possible between characters (left to right) in perception and in memory (e.g., RBNHFD); that is, first a perceptual item, then a memory item, and so forth. Finally, a switching time (ST) is computed, relating the alternate times and the combined pure times for perception and memory.

Of particular interest in this investigation were the derived switching-time scores and their signs. Positive switching times would indicate that it takes time to cycle between mixed perceptual and memory lists, time in excess of that required to process pure perceptual and pure memorial lists. Negative switching times, as sometimes found by Jersild (1927), would suggest that mixed lists take less time to process than pure lists. In the present case, negative switching times might occur if refractory periods are associated with access to pure memory and/or perceptual lists. Alternating between properties or relations might then be more rapid because it would allow the refractory period to dissipate before the next like-item was processed.

In the present experiment, the alternate lists consisted of six items (i.e., three perceptual and three memory items). The memory and perceptual lists consisted of either three items or six items. When three items each were in the memory and perceptual lists, the following switching-time formula was appropriate:

$$
\mathrm{STA}=[A 6-(M 3+P 3)] / 5
$$

The letter-number pairs indicate production times for the associated condition and list length. This formula equates the number of items processed in memory $(M)$, perception $(P)$, and alternate $(A)$ conditions. That is, three items each were always processed from pure memory or perceptual lists, and six items were processed from each alternate list (i.e., memory and perception). Thus, the total response time in the alternate condition was that required to produce six items, whereas the response time in each separate pure condition was that required to produce only three items. The division by five occurs because there are five perception-memory switches in a sixitem sequence of alternations. This formula could have resulted in spuriously large switching times if alternate times were artificially inflated. Alternate times could have been inflated by unexpected changes in list lengths (i.e., three to six or six to three) or by longer start-up or stop times for longer lists. This formula could also have resulted in spuriously small switching times if alternate 
times were artificially reduced. Alternate times could have been reduced, for example, by accelerated performance at the end of the longer lists.

In order to control for such possible confounds in comparisons between perceptual, memory, and alternate production times and in computation of switching times, perceptual and memory lists of Length 6 were also included. The use of six items in these lists resulted in the following switching-time formula:

$$
\mathrm{STB}=[A 6-(M 6+P 6) / 2] / 5
$$

The sum of the pure memory and perception production times is divided by two to mathematically control for the number of characters in the lists. In summary, this experiment provided production times from five conditions: $M 3, P 3, M 6, P 6$, and $A 6$. Two different methods of computing switching time were included to take into account several possible confounding influences and to test the robustness of the switching concept.

\section{Method}

Subjects. The subjects were 14 undergraduates, from psychology classes at Oklahoma State University, who received extra credit for their participation. Their ages ranged from 18 to 43 years.

Stimuli. The stimuli consisted of the first 10 nonconsecutive consonants of the alphabet (B, D, F, H, J, L, N, P, R, and T). The capitalized letters were presented to the subjects on a black-andwhite Sanyo monitor by means of an Apple II+ computer.

Procedure. The experiment was a completely within-subjects design. A sequence of 105 trials was organized into 21 blocks, each block containing one trial from each of the five experimental conditions (i.e., perception and memory at both Lengths 3 and 6 and the alternate condition). Condition order within each block was randomly determined. The letter sequences within each trial were also randomly determined.

After reading an explanation of the types of experimental conditions to be presented, subjects were given one practice trial for each of the five conditions. The stimulus displays for each condition may be seen in Table 1. In the three-letter memory condition, three letters were displayed on the monitor for a 4-sec interval. The subjects were asked to remember the letters in order. Immediately following this display, the screen was cleared, and three asterisks were displayed two lines below the prior display. At this point, a timing clock was started. The subjects' task was to say aloud, as quickly as possible, the three letters they had been asked to remember. Immediately after they finished saying the letters, they were to press the space bar of the computer keyboard. Pressing the space bar ended the trial and stopped the clock, and the production time for that trial was automatically recorded. After a 3-sec interval, the next randomly ordered trial began. The six-letter memory condition was similar, except that the subjects saw six letters in the first display, followed by six asterisks. Again, the subjects' task was to say the letters appearing in the first display as quickly as possible when the asterisks appeared.

In the three-letter perception condition, subjects first saw three asterisks for a 4-sec interval. The screen was then cleared, the timer was started, and a sequence of three letters was displayed two lines below the prior display. The subjects were instructed to read the three letters from the screen as quickly as possible, pressing the space bar when the task was completed. Pressing the space bar stopped the time interval and recorded the production time for that trial. In the six-letter perception condition, the subjects saw six asterisks in the first display, followed by six letters. Subjects were instructed to read the letters as quickly as possible. Again, production times were recorded.
In the alternate condition, memory and perception displays were combined. Three letters were presented in the first display for a 4-sec interval. Subjects were to remember the three letters in order. Following this display, the screen was cleared, the timing interval was started, and a second series of three letters was presented two lines below the previous display. The subjects' task was to alternately say one letter from the perceptual list (which remained on the screen) and then one letter from the memory list. Subjects alternated between perceptual and memory lists until they had said all six letters. Letters in the first display were never duplicated in the second display. A different random assignment of letters to displays was used for each trial.

Each trial was strictly scored for errors during its execution. (A switch connected to the game port was activated by the experimenter to mark an error.) A trial was considered an error if a nonappearing letter was added, if a letter was omitted, or if any letters were said out of sequence.

\section{Results}

The principal results for production time, switching time, and errors are presented in Table 2 . All production and switching times are in milliseconds per list.

Production time. The results are for Blocks 2-21; Block 1 served as practice. The times do not distinguish between correct choices and errors. ${ }^{1}$ For the upper half of the table, the time to alternate for Length 3 was based on six letters. To ensure that comparisons between times in the alternate, perception, and memory conditions were properly weighted, the Length 3 times in the alternate condition were first divided by 2 . For Length 3 , a modes (perception, memory, alternate) $\times$ blocks (2-21) analysis of variance was conducted. A significant main effect of mode was revealed $[F(2,26)=20.96, p<.001]$. Tukey's HSD tests indicated that mean production time was significantly longer in the alternate condition than in the memory and perception conditions. The memory and perception conditions did not differ significantly from each other.

At Length 6, a significant mode (perception, memory, alternate) $\times$ block interaction was revealed $[F(38,494)$ $=1.50, p<.05$ ]. Simple effects tests (Kirk, 1968; Winer, 1971) indicated that the block effect was significant at all modes [smallest $F(19,741)=6.45, p<.05$ ] However, production time differences across blocks were seemingly random and did not reflect an increasing or

Table 2

Means for Production Time (in Milliseconds) Switching Time, and Errors as a Function of List Length and Mode

\begin{tabular}{lrrrrr}
\hline & \multicolumn{2}{c}{ Production Time } & \multicolumn{2}{c}{ Errors (20 Possible) } \\
\cline { 2 - 3 } Condition & \multicolumn{1}{c}{ Mean } & SD & & Mean & SD \\
\hline & & Length 3 & & \\
$M 3$ & 1,446 & 493 & .29 & .61 \\
$P 3$ & 1,688 & 590 & 0 & 0 \\
$A 3 *$ & 2,300 & 775 & $\dagger$ & $\dagger$ \\
STA & 293 & 299 & & \\
& & Length 6 & & \\
M6 & 2,652 & 1,170 & 4.21 & 4.49 \\
P6 & 2,779 & 840 & .07 & .27 \\
A6 & 4,600 & 1,550 & 5.50 & 4.09 \\
STB & 377 & 301 & & \\
\hline
\end{tabular}

*Alternate time divided by 2 . † Same as $A 6$. 
decreasing function. Further simple effects tests revealed a significant mode effect at all blocks [smallest $F(3,520)$ $=20.8, p<.01]$. Tukey's HSD tests indicated that, at all blocks, production time in the alternate condition was longer than in both the perception and memory conditions, which did not differ from each other.

Switching time. Switching times were computed for STA (Equation 1) and for STB (Equation 2). An analysis of variance based on two types of switch $(A, B) \times 20$ blocks indicated that STB (mean $=376.88 \mathrm{msec}$ ) was significantly longer than STA (mean $=293.33 \mathrm{msec}$ ) $[F(19,247)=19.29, p<.001]$. The block effect was not significant $(p>.05)$, indicating that switching between perception and memory was stable over at least a moderate amount of practice.

Error data. As shown in Table 2, the error results across conditions resemble the corresponding production time results. Conditions with high error rates also had large production times, a result contrary to speed-accuracy trade-off at the level of conditions. An analysis of variance conducted on the mean number of errors at each mode and length condition revealed a significant effect of mode. Follow-up tests indicated that there were significantly more errors in the alternate condition than in the perception condition at Length 3 and Length 6 , and in the memory condition at Length 3 . In addition, there were significantly more errors in the memory condition for Length 6 than in either of the perception conditions or in the memory condition for Length 3.

A correlational analysis for production time and the number of errors within each condition indicated that the only significant correlations were positive ones in the memory Length 6 condition $(r=.85, p<.0001)$ and the alternate condition $(r=.62, p<.05)$. These results are contrary to a speed-accuracy trade-off, which would imply a significant negative correlation between errors and production time.

\section{Discussion}

The purpose of this experiment was to develop basic switching-time procedures and to determine the time it takes to switch between memorial and perceptual inputs. Two switching-time formulas were used to compute switching time. Both measures of switching time were large and positive: cycling between perception and memory inputs takes a long time. The mean times involved, STA $=293 \mathrm{msec}$ and STB $=377 \mathrm{msec}$, are of the same order as intensity switching times (Weber et al., 1982). Finally, the switching-time effect also seems to be robust in the face of at least moderate practice over 20 blocks.

STB was significantly larger than STA. This difference must be due to the disproportionately faster times per character in the six-item memory and perception lists than in the three-item lists, because the alternate production time used in the switching-time formula for Length 6 was the same as that for the formula for Length 3 . The longer Length 6 perception and memory lists may have been produced proportionally faster, because start-up and stop times are probably relatively long in comparison to times for producing characters. Therefore, the six-item perception and memory lists actually take less time per character than do the three-item lists.

Which is the better switching-time index, A or B? STA seems to be better for our purposes for two reasons: it is (1) more conservative and (2) more flexible. As an illustration of flexibility, the memory Length 6 condition used in computing STB begins to press the limits of memory span and is afflicted with a moderately high error rate. Thus, if switching time is to be investigated for variables such as list length, STA allows for a wider range of variation before memory span is exceeded.

One source of concern was whether the 4-sec duration of the first display was sufficient for memory encoding. The nonsignificant production time difference between the memory and perception conditions at Length 6 alleviates this concern, because the perception conditions allowed unlimited viewing. If anything, memory production times were faster than perceptual readouts, although the difference was not significant. Finally, the production time results were not due to speed-accuracy trade-off, since the usual finding with list processing occurred: total errors and time were either uncorrelated or positively correlated.

\section{EXPERIMENT 2}

Recent work by Baddeley (1982) has suggested that working memory is partitioned into three parts: (1) an articulatory phonetic loop or scratch pad, (2) a visual scratch pad, and (3) control processes. If this partitioning idea is correct, then an important issue is the extent to which the items stored in the articulatory and visual scratch pads share the limited capacity of short-term memory with the control processes. The switching-time paradigm may be useful for determining the extent to which storage and control are separate, nonoverlapping processes. For example, the length of perceptual and/or memory lists could be increased to determine effects on the control processes used in perception-memory attention switching. Several theories offer predictions as to how this manipulation would affect control processing.

First, a separate channel or module type of theory (Broadbent, 1958; Fodor, 1983) would propose that there are separate stores for items and for control-process programs in working memory. If items and control processes are stored separately, or informationally encapsulated, they should be relatively independent of one another. That is, variations in the number of items in memory/perception lists should not affect the control processes involved in alternation. Switching times and accuracy levels should be constant across different list lengths. Second, a flexible capacity explanation (Johnston \& Heinz, 1978; Kahneman, 1973; Logan, 1980; Moray, 1967; PascualLeone, 1970) would propose that information processing resources are shared between item and control-process 
space. Therefore, an increase in memory and perceptual load would decrease the capacity available for control processes. Hence, control-process components might be loaded piecemeal, perhaps as successive program overlays. As a result, switching time should be an increasing function of list length.

This experiment was designed to determine the effect of list length on switching processes. The methodology from Experiment 1 was repeated. However, the number of items in the memory and perceptual lists varied from 2 to 5 , while those in the alternate lists varied from 4 to 10 .

\section{Method}

Subjects. The subjects were 14 undergraduates, from psychology classes at Oklahoma State University, who received extra credit for participation. Seven males and 7 females were involved in the experiment; their ages ranged from 18 to 27 years.

Procedure. The experiment was a 3 (modes) $\times 4$ (list lengths $2,3,4,5$ ) within-subjects design. As in Experiment 1, the modes were perception, memory, and alternate. The stimuli within each mode and list length condition were presented exactly as they were in Experiment 1. The trial sequences were organized into 11 blocks, so that each block contained one trial from each of the 12 possible conditions (i.e., P2-P5, M2-M5, A2-A5). The condition order within each block was randomly determined. The letter sequences within each trial were also randomly determined.

The formula used to compute switching time was a general case of STA from Experiment 1:

$$
\begin{aligned}
\text { STA }= & {[\text { Alternate. } 2 L-(\text { Memory } . L+\text { Perception. } L)] / } \\
& (2 L-1)
\end{aligned}
$$

The only aspect different from Equation 1 is that $L$, the length of the list, is now a variable. Thus, $2 L-1$ is the number of switches in an alternating sequence consisting of a perception list, P. $L$, and a memory list, M.L. For example, the number of switches for Length 3 was five (three perception items plus three memory items minus one).

Each trial was scored for errors during its execution. A trial was considered an error if a nonappearing letter was added, if a letter was omitted, or if any letters were listed out of sequence. Note that this is a very strict criterion: any error results in treating all the data for that trial as an error.

\section{Results}

A description of the data is presented in Table 3. The alternate scores for Table 3 were weighted by 0.5 so that production time scores for each mode would be based on an equal number of characters. All analyses of variance were conducted on scores so adjusted. Block 1 data and all trials involving error production times were excluded from analysis. The remaining data were analyzed in terms of mean production time across blocks.

Production time. The principal results are shown in Table 3. A modes $\times$ lengths analysis of variance revealed a significant interaction $[F(6,72)=30.88, p<.0001]$. Simple effects tests indicated that mode was significant at all lengths [smallest $F(2,96)=3.05, p<.05$ ]. Tukey's HSD tests indicated that production time in the alternate condition was significantly longer than production time in both the memory and perception conditions for Lengths 3,4 , and 5. Perception and memory conditions did not
Table 3

Means for Production Time (in Milliseconds), Switching Time,

\begin{tabular}{|c|c|c|c|c|}
\hline \multirow[b]{2}{*}{ Condition } & \multicolumn{2}{|c|}{ Production Time } & \multicolumn{2}{|c|}{ Errors (10 Possible) } \\
\hline & Mean & SD & Mean & $\overline{S D}$ \\
\hline \multicolumn{5}{|c|}{ Length 2} \\
\hline$M 2$ & 1,109 & 228 & 0 & 0 \\
\hline$P 2$ & 1,198 & 213 & .07 & .27 \\
\hline$A 2 *$ & 1,320 & 717 & .79 & .89 \\
\hline ST2 & 120 & 214 & & \\
\hline \multicolumn{5}{|c|}{ Length 3} \\
\hline$M 3$ & 1,381 & 233 & .07 & .27 \\
\hline$P 3$ & 1,505 & 299 & 0 & 0 \\
\hline$A 3^{*}$ & 1,911 & 745 & 1.86 & 1.51 \\
\hline ST3 & 176 & 128 & & \\
\hline \multicolumn{5}{|c|}{ Length 4} \\
\hline$M 4$ & 1,606 & 322 & .21 & .58 \\
\hline P4 & 1,911 & 337 & .07 & .27 \\
\hline$A 4 *$ & 2,903 & 1,092 & 4.86 & 2.82 \\
\hline ST4 & 334 & 125 & & \\
\hline \multicolumn{5}{|c|}{ Length 5} \\
\hline M5 & 2,037 & 384 & 1.00 & 1.71 \\
\hline P5 & 2,168 & 435 & .07 & .27 \\
\hline$A 5^{*}$ & 3,600 & 1,698 & 7.29 & 1.59 \\
\hline ST5 & 336 & 183 & & \\
\hline
\end{tabular}
and Number of Errors as a Function of List Length and Mode

*Alternate times divided by 2 .

differ from each other. None of the conditions differed from each other for Length 2 .

Further simple effects tests indicated that the effect of length was significant at all modes [smallest $F(3,108)=$ 105.07, $p<.01$ ]. Tukey's HSD tests indicated that in the memory condition, production time at Length 5 was greater than production time at Lengths 2,3 , and 4 . In the perception condition, production times at Lengths 5 and 4 were longer than those at Lengths 2 and 3 . Production time at Length 3 was longer than that at Length 2. In the alternate condition, all of the pairwise comparisons were significant. Production time at Length 5 was longer than those at Lengths 2, 3, and 4. Production time at Length 4 was greater than those at Lengths 2 and 3 . Finally, production time at Length 3 was greater than that at Length 2.

Switching time. Mean switching times for each length are shown in Table 3. (Alternate times were not divided by two for the switching-time results because the proper weighting is given by the switching-time formula.) The switching time for each length was computed according to Equation 3. A one-way analysis of variance revealed a significant effect of length $[F(3,36)=13.09$, $p<.0001]$. Tukey tests indicated that times per switch for Length 5 and Length 4 were significantly longer than those for Length 3 and Length 2 . The switching time for Length 5 may have been artificially low, because it was based on a relatively small number of correct trials. As shown in Table 3, for the alternate Length 5 condition, at least one error occurred on an average of 7.29 of the 10 trials. 
Error data. Error data were analyzed in terms of the number of errors in each mode $x$ length condition. The mean number of errors in each condition is indicated in Table 3. A modes $\times$ lengths analysis of variance revealed a significant interaction $[F(6,72)=30.78, p<.0001]$. Tests of simple effects indicated that mode effects were significant at all lengths [smallest $F(4,96)=798.35$, $p<.001]$. Tukey tests $(\alpha \leq .05)$ indicated that at all lengths there were significantly more errors in the alternate condition than in the memory and perception conditions, which did not differ from each other. Further simple effects tests indicated that the effects of length were significant in the memory and alternate conditions only. In the memory condition, significantly more errors were made at Length 5 than at all other lengths. In the alternate condition, more errors were made at Length 5 than at any of the other lengths, and at Length 4 than at Lengths 3 and 2. Correlational analyses within conditions, for production times and errors, failed to reveal any significant correlation coefficients at the .05 level, belying a speed-accuracy trade-off. Between conditions, the error data paralleled the production time data (Table 3).

\section{Discussion}

The results of Experiment 2 suggest that the length of the list has a fairly regular increasing effect on production time for each mode. The nature of the increase is such that switching time is an increasing function of list length. Thus, it is possible to eliminate one model of working memory structure. Item representation and controlprocess representation do not partake of completely separate channels; if they did, this function would be flat. The remaining alternative, that control processes and item memory share capacity, is strongly supported by the increasing function of switching time and list length.

\section{GENERAL DISCUSSION}

Two experiments were conducted to investigate attention switching between perception and memory. In Experiment 1 , methodology and procedures were developed. It was demonstrated that attention switching is a timeconsuming process. The results are consistent, therefore, with those of previous experiments in which timeconsurning attention switches were demonstrated (Jersild, 1927; LaBerge, 1973; Treisman, 1960; Weber et al., 1982). In Experiment 2, item and control-process representation involved in attention switching was explored. The results are consistent with capacity theories of attention which would predict that items stored share limited attentional capacity with control processes (e.g., Kahneman, 1973).

According to Kahneman's (1973) capacity theory, an allocation policy determines how much of one's available attentional capacity is directed to various activities involved in tasks. If the supply of attention does not meet task demands, the level of performance declines. Thus, Kahneman's model stressed ways in which the capacity demands of various tasks affect performance on those tasks. The results from the present investigation suggest that excessive capacity demands can also affect the processes which control the allocation of attention to tasks. That is, extreme capacity demands not only lead to declines in task performance, but they can also slow the control processes that set the allocation policy. In the memory-perception switching task, these effects were manifested by increased production time in the alternate condition and by significant length effects on switching time. Increases in the number of items processed appeared to slow resetting of the allocation policy that determined whether attention would be switched to perceptual or memorial processes. This finding is contrary to a modularity or information encapsulation view (Fodor, 1983). It is consistent, however, with Logan's (1980) findings that item memory and S-R mapping rules compete for shortterm memory capacity. An interesting conjecture is that item memory, mapping rules, and all control processes share capacity.

As suggested by reviewers, it is appropriate to consider possible rival interpretations to our findings, interpretations that do not involve switching between perception and memory.

\section{The Stroop Interpretation}

Is it possible that what has been referred to as a switching between perception and memory is actually a Strooplike interference (Dyer, 1973) in which letters in perception interfere with access to letters in memory, or vice versa? We believe that this is not the case for the following reasons. First, there are no obvious properties, such as color names and colors, in conflict here. The letter sets to be alternated between are in different "places," shortterm memory and the perceived display, and on a given trial these two sets do not overlap. Second, looking and recall are sufficiently different that it is unlikely that they would involve competition for the same components. Third, it is not clear how a Stroop-like interpretation would handle the effect of list length on switching time. Fourth, whatever other processes one may wish to posit, it is clear that to perform the task, there must be some kind of switching between the perception and memory lists. If that is the case, then the supposition of other processes is multiplying explanatory entities beyond immediate necessity. Fifth, it is possible to turn the argument completely around: switching may be a more fundamental explanation than interference, and in fact, the Stroop interference effect may be due to spontaneous switching of ascendancy between color processing and text processing. For these reasons, it is unlikely that the present results can be explained by Stroop-like interference.

\section{Switching Between Memory Chunks}

Could the present results be explained on the basis of switching between any two sets of letters, perhaps with both sets in memory rather than a specific perception- 
memory switch? First, there is no doubt that there can be substantial switching times between chunks of items in memory. Weber et al. (1982) had a condition involving switching between letter and digit sets, both of which were in memory. At first moderate switching times occurred, but with practice on the same sets, the switching times approached zero. Thus, the issue is not whether switching can also occur between separate chunks in memory, but whether the present results can be so explained. We believe they cannot for the following reasons. First, subjects were instructed to read the letters. They looked like they were doing so while alternating between the perception and memory lists, and reading would seem to be much easier than memorizing the perceptual set. Second, if subjects encoded in memory the entire perceptual set in the alternation condition, would they also not do so in the pure perceptual condition? If so, the pure perception and the pure memory data should have been equivalent. Although this was approximately the case for time and errors, it was not the case for list length. There was a list length effect for memory, but not for perception, indicating that equivalent forms of processing were not occurring. Third, the longer length conditions, Lengths 4 and 5 , would probably exceed short-term memory capacity in the alternate conditions, because there would be 4(5) perceptual items plus 4(5) memory items. Thus if the nominally perceptual items were also memory encoded, this would produce a combined memory set of $8(10)$ items, total. Such a memory load would be very difficult in comparison to reading the perceptually presented list, as instructed. For these reasons, we believe that the switching that occurred was, in fact, between perceptual and memory lists.

\section{A Model of Switching Between Perception and Memory}

A preliminary model of perception-memory switching is now proposed. It is based substantially upon the work of Baddeley (1982). The task seems to require processes analogous to those of a computer that accesses several subroutines. First, subjects assemble instructions for each condition. These instructions remain accessible during the task (Pascual-Leone, 1970). On each trial, subjects then use the nature of the two stimulus displays (i.e., letters or asterisks) to decide which set of instructions or which subroutine is appropriate.

For example, subjects can decide that a given trial is a perception trial, if the first display is asterisks. Attention would then be allocated to a perception subroutine, which would involve encoding letters from the second display into the visual scratch pad, saying them sequentially, and then pressing the space bar. In this experiment, the perception subroutine appeared to be slowed by list lengths of four and five items. These lists were produced more slowly than, although as accurately as, lists of Lengths 2 and 3.

In contrast, if the first display is letters, then the subject must wait for the second display before attention can

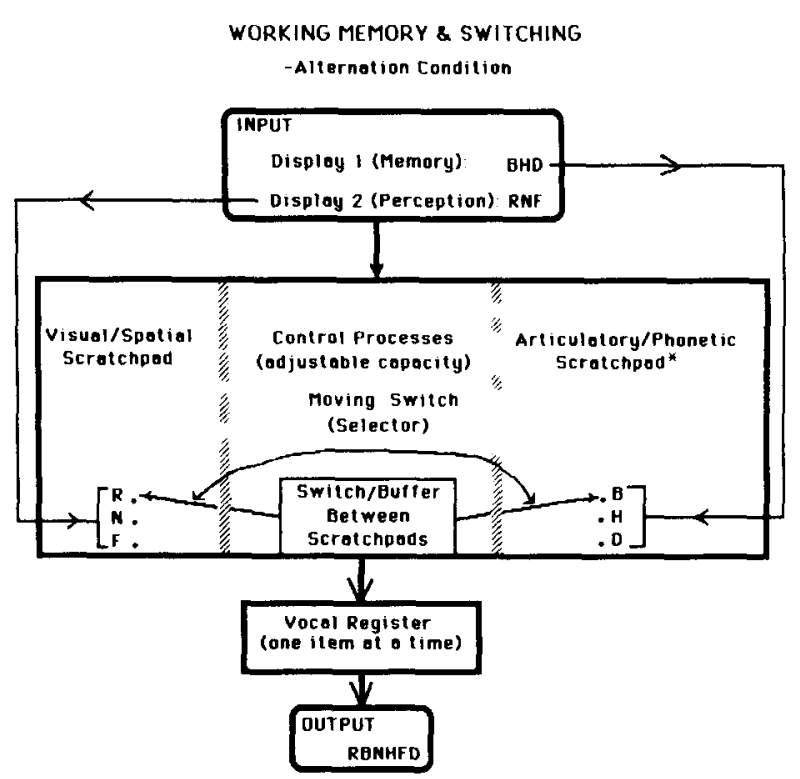

Figure 1. Hypothesized working memory processes involved in perception-memory attention switches. The asterisk indicates information that has already been transferred from the visual scratch pad.

be correctly allocated to an appropriate subroutine. If the second display is asterisks, attention would be allocated to a memory subroutine. This subroutine would involve the serial retrieval of items previously stored in an articulatory/phonetic scratch pad, saying the items, and pressing the space bar. In this experiment, the memory subroutine was slowed and produced more errors for lists of Length 5 compared to those of Lengths 2, 3, and 4.

Finally, if both the second and first displays were letters on a given task, attention would be allocated first to the perception subroutine, then to the memory subroutine. This cycling process would continue until all letters were produced. Figure 1 illustrates the hypothetical process.

The subjects must keep track of which list is to be currently accessed, and which items in each list have already been produced. Both operations would be accomplished through the position of the switch illustrated in the figure. In Experiment 2, alternation processing became longer and produced more errors for each new item added to the memory and perception lists. Switching time was longer for lists of Lengths 4 and 5 than for lists of Lengths 2 and 3 . These results suggest, then, that increases in the number of items to be processed not only affected performance on the task (time and accuracy), but also affected the control processes themselves. Attentional capacity appeared to be overloaded when the subjects were required to alternate between the longer lists, slowing the attentional allocation policy. The heavy broken lines in the figure on each side of the control-process section indicate adjustable capacity: as the number of items in the visual/spatial and articulatory/phonetic scratch pads increases, there is a constriction of available capacity for the use of control processes. As capacity diminishes for 
control-process functioning, it may be necessary to load only part of the necessary routines into working memory at a time; that is, successive overlays are required. The model in Figure 1 also suggests why the alternate condition takes much longer to process than either of the pure conditions, memory and perception. It is much more complex to switch modes (alternate) than to change items (memory, perception).

In summary, the results of this investigation suggest that it takes time to switch or reallocate attention between perceptual and memorial inputs. A model suggesting that attention switching is analogous to computer access of subroutines was proposed. The fact that switching time and errors increased as a function of list length suggests that the subroutines cannot be accessed quickly and efficiently when attentional capacity is overloaded. Item load appears to affect control processes that set allocation policies as well as one's ability to perform memory and/or perceptual tasks.

\section{REFERENCES}

BADDELEY, A. (1982). Reading and working memory. Bulletin of the British Psychological Society, 35, 414-417.

Broadbent, D. E. (1958). Perception and communication. New York: Pergamon Press.

BYRD, D. M., \& GHOLSON, B. (1984). A cognitive-developmental model of reading. In B. Gholson \& T. L. Rosenthal (Eds.), Application of cognitive-developmental theory. New York: Academic Press.

DYER, R. N. (1973). The Stroop phenomenon and its use in the study of perceptual, cognitive, and response processes. Memory \& Cognition, 1, 106-120.

FoDor, J. A. (1983). The modularity of mind. Cambridge, MA: The MIT Press.

JersiLd, A. T. (1927). Mental set and shift. Archives of Psychology (Whole No. 89)

JoHnston, W. A., \& HeINZ, S. P. (1978). Flexibility and capacity demands of attention. Journal of Experimental Psychology: General, $107,420-435$.
Kahneman, D (1973). Attention and effort. Englewood Cliffs, NJ: Prentice-Hall.

KIRK, R. E. (1968). Experimental design: Procedures for the behavioral sciences. Monterey, CA: Brooks/Cole.

LABERGE, D. (1973). Identification of two components of the time to switch attention: A test of a serial and a parallel model of attention. In S. Kornblum (Ed.), Attention and performance $\mathrm{I}$. New York: Academic Press.

LOGAN, G (1980). Short-term memory demands of reaction-time tasks that differ in complexity. Journal of Experimental Psychology: Human Perception \& Performance, 6, 375-389.

Moray, N. (1967). Where is capacity limited? A survey and a model. Acta Psychologica, 27, 84-92.

Pascual-Leone, J. A. (1970). Mathematical model for the transition rule in Piaget's developmental stages. Acta Psychologica, 32, 301-345.

Shulman, G. L., Remington, R. W., \& McLean, J. P. (1978). Moving attention through visual space. Journal of Experimental Psychology: Human Perception \& Performance, 5, 522-526.

SPECTOR, A., \& Biederman, I. (1976). Mental set and mental shift revisited. American Journal of Psychology, 89, 669-679.

Treisman, A. M (1960). Contextual cues in encoding listening. Quarterly Journal of Experimental Psychology, 12, 342-348.

Weber, R. J., Blagowsky, J., \& Mankin, R. (1982). Switching time between overt and covert speech: Generative attention. Memory \& Cognition, 10, 546-553

WINER, B. J. (1971). Statistical principles in experimental design. New York: McGraw-Hill.

\section{NOTE}

1. Due to a programming error, production times for correct and incorrect responses were not separately stored. However, total errors per subject per condition were accumulated. In order to eliminate effects due to outlying observations, the analyses of variance were conducted on truncated data. For each subject, within each condition, production times greater than or less than two standard deviations away from the mean were replaced by the mean.

(Manuscript received May 22, 1985; revision accepted for publication December 13, 1985.) 